\title{
Augmented cartilage regeneration by implantation of cellular versus acellular implants after bone marrow stimulation: a systematic review and meta-analysis of animal studies
}

\author{
Michiel W Pot ${ }^{\text {Corresp., }}{ }^{1}$, Toin H van Kuppevelt ${ }^{1}$, Veronica K Gonzales ${ }^{2}$, Pieter Buma ${ }^{2}$, Joanna IntHout ${ }^{3}$, Rob \\ BM de Vries ${ }^{4}$, Willeke F Daamen ${ }^{\text {Corresp. } 1}$ \\ 1 Department of Biochemistry, Radboud Institute for Molecular Life Sciences, Radboud university medical center, Nijmegen, The Netherlands \\ 2 Department of Orthopedics, Radboud Institute for Molecular Life Sciences, Radboud university medical center, Nijmegen, The Netherlands \\ 3 Department for Health Evidence, Radboud university medical center, Nijmegen, The Netherlands \\ 4 SYRCLE (SYstematic Review Centre for Laboratory animal Experimentation), Central Animal Laboratory, Radboud university medical center, Nijmegen, \\ The Netherlands \\ Corresponding Authors: Michiel W Pot, Willeke F Daamen \\ Email address: Michiel.Pot@radboudumc.nl, Willeke.Daamen@radboudumc.nl
}

Bone marrow stimulation may be applied to regenerate focal cartilage defects, but generally results in transient clinical improvement and formation of fibrocartilage rather than hyaline cartilage. Tissue engineering and regenerative medicine strive to develop new solutions to regenerate hyaline cartilage tissue. This systematic review and metaanalysis provides a comprehensive overview of current literature and assesses the efficacy of articular cartilage regeneration by implantation of cell-laden versus cell-free biomaterials in the knee and ankle joint in animals after bone marrow stimulation. PubMed and EMBASE (via OvidSP) were systematically searched using tissue engineering, cartilage and animals search strategies. Included were primary studies in which cellular and acellular biomaterials were implanted after applying bone marrow stimulation in the knee or ankle joint in healthy animals. Study characteristics were tabulated and outcome data were collected for meta-analysis for studies applying semi-quantitative histology as outcome measure (117 studies). Cartilage regeneration was expressed on an absolute 0 $100 \%$ scale and random effects meta-analyses were performed. Implantation of cellular biomaterials significantly improved cartilage regeneration by $18.6 \%$ compared to acellular biomaterials. No significant differences were found between biomaterials loaded with stem cells and those loaded with somatic cells. Culture conditions of cells did not affect cartilage regeneration. Cartilage formation was reduced with adipose-derived stem cells compared to other cell types, but still improved compared to acellular scaffolds. Assessment of the risk of bias was impaired due to incomplete reporting for most studies. Implantation of cellular biomaterials improves cartilage regeneration compared to acellular biomaterials. 
1 Augmented cartilage regeneration by implantation of cellular versus acellular implants after bone marrow stimulation: a systematic review and meta-analysis of animal studies

4 Michiel W. Pot ${ }^{1}$, Toin H. van Kuppevelt ${ }^{1}$, Veronica K. Gonzales ${ }^{2}$, Pieter Buma ${ }^{2}$, Joanna IntHout ${ }^{3}$, 5 Rob B.M. de Vries ${ }^{4}$, Willeke F. Daamen ${ }^{1 *}$

6

71 Department of Biochemistry, Radboud Institute for Molecular Life Sciences, Radboud 8 university medical center, Nijmegen, The Netherlands

92 Department of Orthopedics, Radboud Institute for Molecular Life Sciences, Radboud 10 university medical center, Nijmegen, The Netherlands

113 Department for Health Evidence, Radboud university medical center, Nijmegen, The 12 Netherlands

134 SYRCLE (SYstematic Review Centre for Laboratory animal Experimentation), Central Animal 14 Laboratory, Radboud university medical center, Nijmegen, The Netherlands

*Corresponding author.

Willeke Daamen PhD

19 Radboud Institute for Molecular Life Sciences, Radboud university medical center, Nijmegen,

20 The Netherlands

E: Willeke.Daamen@radboudumc.nl, T: +31 243610557 
22 Abstract

23 Introduction: Bone marrow stimulation may be applied to regenerate focal cartilage defects,

24 but generally results in transient clinical improvement and formation of fibrocartilage rather

25 than hyaline cartilage. Tissue engineering and regenerative medicine strive to develop new

26 solutions to regenerate hyaline cartilage tissue. This systematic review and meta-analysis

27 provides a comprehensive overview of current literature and assesses the efficacy of articular

28 cartilage regeneration by implantation of cell-laden versus cell-free biomaterials in the knee and ankle joint in animals after bone marrow stimulation.

Methods: PubMed and EMBASE (via OvidSP) were systematically searched using tissue engineering, cartilage and animals search strategies. Included were primary studies in which cellular and acellular biomaterials were implanted after applying bone marrow stimulation in the knee or ankle joint in healthy animals. Study characteristics were tabulated and outcome data were collected for meta-analysis for studies applying semi-quantitative histology as outcome measure (117 studies). Cartilage regeneration was expressed on an absolute $0-100 \%$ scale and random effects meta-analyses were performed.

Results: Implantation of cellular biomaterials significantly improved cartilage regeneration by $18.6 \%$ compared to acellular biomaterials. No significant differences were found between biomaterials loaded with stem cells and those loaded with somatic cells. Culture conditions of cells did not affect cartilage regeneration. Cartilage formation was reduced with adiposederived stem cells compared to other cell types, but still improved compared to acellular scaffolds. Assessment of the risk of bias was impaired due to incomplete reporting for most 43 studies.

44 Conclusion: Implantation of cellular biomaterials improves cartilage regeneration compared to 45 acellular biomaterials. 


\section{Introduction}

Articular cartilage facilitates joint loading and movement by resisting compressive and shear forces [1]. For patients, localized cartilage defects can have detrimental long term effects such as joint dysfunction, pain, and degenerative osteoarthritis. Upon cartilage damage, its avascular nature prevents spontaneous healing [2]. Clinical treatments for full-thickness cartilage defects and osteochondral lesions include bone marrow stimulation techniques, e.g. microfracture and subchondral drilling, and autologous chondrocyte implantation. Defect size generally determines treatment, where microfracture and autologous chondrocyte implantation are used to treat small $\left(<2.5 \mathrm{~cm}^{2}\right)$ and large lesions $\left(>2.5 \mathrm{~cm}^{2}\right)$, respectively [3]. Microfracture surgery is a minimally invasive and inexpensive one-step approach, where multiple perforations, microfractures, are made in the subchondral bone plate to induce bleeding and provoke a reparative response. The formed blood clot consists of bone marrow-derived mesenchymal stem cells (BM-MSCs), growth factors and other proteins, supporting cartilage formation [4]. The repaired tissue, however, generally consists of fibrous cartilage, which lacks the mechanical properties of native hyaline cartilage [5]. Microfracture results in temporary clinical improvement only [6], and the demand for improved cartilage regeneration persists.

Cartilage regeneration may be improved by tissue engineering and regenerative medicine (TERM) in addition to bone marrow stimulating techniques. TERM encompasses the development of biomaterials, which can be loaded with cells and biologics [7]. Upon implantation and infiltration of BM-MSCs, the biomaterial may act as a template to guide/stimulate cartilage regeneration [3]. In a previous systematic review and meta-analysis on animal models, we showed that acellular biomaterials in addition to bone marrow stimulation was more effective in regenerating cartilage in vivo than bone marrow stimulation alone, which was further improved by use of biologics [8].

When biomaterials are loaded with cells, bone marrow stimulation may be even more effective. Biomaterials loaded with cells after bone marrow stimulation has been widely investigated in vivo, and included loading of chondrocytes [9-11], BM-MSCs [12-14], synoviumderived mesenchymal stem cells (SD-MSCs) [15-17], adipose-derived stem cells (ADSCs) [18-20], periosteal cells [21, 22], fibroblasts [23], umbilical cord stem cells (UCSC) [23, 24] and 
75 embryonic stem cells (ESC) [25]. Cells are either used directly after harvesting [26, 27] or after 76 an additional in vitro step of cell expansion [28, 29] and/or differentiation [30, 31].

77 In this systematic review and meta-analysis, we present a comprehensive overview of all 78 current literature regarding regeneration of articular cartilage by implantation of cell-laden 79 versus cell-free biomaterials in the knee and ankle joint after bone marrow stimulation in 80 animal models (Fig. 1). We further investigated the effect of loading biomaterials with (1) stem 81 cells versus somatic (differentiated) cells, (2) different cell types (e.g. chondrocytes, MSCs, 82 ADSCs), and (3) culture conditions of cells (e.g. use after harvesting, in vitro expansion and/or 83 differentiation). In the meta-analysis, histological scores from semi-quantitative histological 84 scoring systems were used to assess the effect on cartilage regeneration. 
85

86

87

88

89

90

91

92

93

94

95

96

97

98

99

\section{Materials and Methods}

\subsection{Search strategy}

An extensive literature search was performed in PubMed and EMBASE (via OvidSP) to identify relevant peer-reviewed articles until June 29, 2016, using methods defined by de Vries et al. [32] and Leenaars et al. [33]. The search strategy (Supplementary Information 1) consisted of search components for tissue engineering [34] and cartilage [8]. Results were refined for animal studies by applying animal search filters $[35,36]$. No language restrictions were applied.

\subsection{Study selection}

After obtaining all references, duplicates were manually removed in EndNote X7 (Thomson Reuters, Philadelphia, Pennsylvania) by one author (MP). Resulting references were screened for relevance by two independent authors (MP and VG/WD) based on title, title/abstract and full-text using Early Review Organizing Software (EROS, Institute of Clinical Effectiveness and Health Policy, Buenos Aires, Argentina, www.eros-systematic-review.org). In case of disagreement between authors or any doubt, references were included for further screening. An overview of all exclusion criteria per screening phase is provided in Supplementary Information 2.

Studies were included for risk of bias assessment and meta-analysis when semiquantitative histological scoring was used as outcome measure.

\subsection{Study characteristics}

Study characteristics were extracted from the studies by MP. Basic information (author, year of publication), animal model characteristics (species, strain, sex, etc.), experimental characteristics (surgery, biomaterial, follow-up, etc.), cell characteristics (cell type, culture conditions, etc.) and outcome characteristics (macroscopic evaluation, histology and semiquantitative histological scoring, etc.) were obtained.

\subsection{Risk of bias assessment}

The methodological quality was assessed for studies included in the meta-analysis. A risk of bias analysis was performed according to an adapted version [8] of the tool described by Hooijmans 
115 et al. [37]. Selection, performance, detection and attrition bias were scored independently by

$116 \mathrm{MP}$ and VG/WD using questions and a flowchart [8], where '-', '?' and '+', indicating low,

117 unknown and high risk of bias. In case of differences between authors, results were discussed

118 until consensus was reached. Unfortunately, 16 articles were published in Chinese and we did

119 not have the resources to obtain certified translations of these articles. We were, however, able

120 to successfully extract the data of these studies using Google Translate and used the data in the

121 meta-analysis. A sensitivity analysis was performed to evaluate the effect of language

122 (exclusion of Chinese articles, see 2.5.2).

\section{$124 \quad 2.5 \quad$ Analysis preparations and meta-analysis}

\section{2.5.1 Analysis preparations}

126 Meta-analyses were performed for outcome measure semi-quantitative histology; data were 127 used from studies that compared biomaterials with (experimental group) and without cells 128 (control group). In general, these histological scoring systems and their components, 129 extensively reviewed by Rutgers et al. [38], evaluate the degree of cartilage regeneration by 130 scoring parameters like Safranin-O staining (which stains negatively charged 131 glycosaminoglycans, an important component of cartilage tissue), surface integrity and cartilage 132 thickness.

133 Outcome data (mean, standard deviation (SD) and number of animals) were extracted from the 134 studies for all time points as follows: 1) numerical data from the text/tables, 2) graphical results 135 by measuring the mean and SD using ImageJ (1.46r, National Institutes of Health USA), 3), 136 boxplot results by recalculating from median, range and sample size to mean and SD [39], and

137 4) for results presented as mean and confidence interval ( $\mathrm{Cl}$ ) per group, the following equation

138 was used to recalculate $\mathrm{Cl}$ to a standard deviation: $S D=\sqrt{N} \times \frac{\text { upper limit - lower limit }}{3.92}$ for a $95 \% \mathrm{Cl}$

139 [40]. When data were missing or unclear, authors were contacted to provide data. Studies were 140 excluded from meta-analysis in case data could not be retrieved or remained unclear (e.g. 141 missing SD, all SD's similar to corresponding mean, and histological scores exceeding 142 maximum), unless data were sufficiently clear to make assumptions (i.e. group size and number 143 of animals per time point and analyses, see Supplementary Information 3). A sensitivity analysis 
144 was performed to evaluate the effect of assumptions (exclusion of articles with assumptions, 145 see 2.5.2). Histological scoring systems describe the degree of cartilage regeneration with 146 different scoring scales. To compare data from different studies, all data were converted to a $147100 \%$ cartilage regeneration scale by dividing both the mean and SD by the maximum score of 148 the scoring system and multiplying the outcome by $100 \%$. In this systematic review, healthy 149 tissue is represented as $100 \%$ cartilage regeneration. Lower percentages indicate less 150 regenerated cartilage. When results of experimental groups could be combined per study (i.e. 151 outcome of various biomaterials seeded with one cell type), we did so, followed the approach 152 described in the Cochrane Handbook, table 7.7 [40], which means that we calculated a 153 weighted average of the results with an appropriate standard deviation. Time points of 154 treatment groups were combined using the same approach. The mean and corresponding standard error (SE) per treatment group were subsequently calculated per study.

\subsubsection{Meta-analysis}

158 The main research question was: Is there an overall beneficial effect on cartilage regeneration of implanting biomaterials loaded with cells compared to acellular biomaterials?

We used a bivariate approach to model a random effects meta-analysis, i.e. separate outcomes for the control and experimental group were used with their respective SEs. The correlation between these two outcomes was modeled with a compound symmetry covariance matrix, as this resulted in a lower Akaike Information Criterion value than an unstructured covariance matrix.

To evaluate the effect of specific variables on treatment outcome for the experimental group (biomaterials loaded with cells), the following sub-questions were addressed: 1) Is there a difference between the use of stem cells and somatic (differentiated) cells (stem cells vs. somatic cells)?; 2) Do differences among various cell subgroups exist (e.g. chondrocytes vs. other cells)?; 3) Is there a difference between biomaterials loaded with cells which were not cultured in vitro, were expanded in vitro or were differentiated in vitro (during surgery vs. expansion, surgery vs. differentiation, and expansion vs. differentiation)? Results are depicted as \% cartilage regeneration $(95 \% \mathrm{Cl}$ : [lower $\mathrm{Cl}$, upper $\mathrm{Cl}$ ]. The mean difference $(\%[95 \% \mathrm{Cl}]$ ) is 
173 presented as condition A - condition B. Based on a previous study, data of all time points were 174 used [8]. Subgroup analyses were performed in case subgroups consisted of more than five 175 experimental groups in at least 3 studies. Most studies contained more than one experimental 176 group, therefore the total number of studies and number of experimental groups (no. of 177 studies/groups) is provided in the analysis. No adjustment for multiple testing was applied in analyses of sub-questions.

Sensitivity analyses were performed on the main research question to evaluate the effect of language (excluding Chinese articles, as the risk of bias for these articles was not investigated), and the effect of assumptions (excluding articles for which assumptions were made) in the meta-analysis.

SAS/STAT ${ }^{\circledR}$ software version 9.2 for Windows, copyright@ 2002-2008 by SAS Institute Inc., Cary, NC, USA, was used to perform statistical analyses. R software version 3.0.1 [41] with package meta [42] was used to create the funnel plot, which illustrates effect sizes of all studies versus their precision, and test for the asymmetry, using the method of moments estimator for the between study variation [43]. $I^{2}$ was used as a measure of heterogeneity. $I^{2}$ measures the percentage of variability in treatment effect estimates that is due to between study heterogeneity rather than chance [44]. If $\mathrm{I}^{2}$ is $0 \%$, this suggests that the variability in the study estimates is entirely due to chance. If $\mathrm{I}^{2}$ is $>0 \%$ there might be other reasons for variability. ReviewManager (RevMan, Version 5.3. Copenhagen: The Nordic Cochrane Centre, The Cochrane Collaboration, 2014) was used to create the forest plot. 


\section{Results}

\section{$194 \quad 3.1$ Search and study inclusion}

195 Searching PubMed and EMBASE databases for references regarding cartilage regeneration by implantation of cellular and acellular biomaterials in the knee and ankle joint in combination with bone marrow stimulation resulted in a total of 11,248 references (Pubmed 4,743, Embase 6,505). Removal of duplicates left 7,354 references. Screening by title and title/abstract resulted in exclusion of 6,744 references. Full-text of 610 studies resulted in 146 included studies. The full-text of some studies [45-47] could not be retrieved and these were excluded.

In the meta-analysis, studies were used which applied semi-quantitative histology as outcome measure, resulting in 117 included studies. A risk of bias assessment (Fig. 2) was performed for 101 of 117 studies (excluding Chinese studies). Supplementary Information 3 provides an overview of all included studies after full-text screening, risk of bias assessment and meta-analysis, as well as detailed information regarding reasons for exclusion and assumptions made for certain studies. Supplementary Information 4 contains the reference list and abbreviations of Supplementary Information 3 studies.

\subsection{Study characteristics}

210 A large variation between studies was observed regarding animal model characteristics

211 (species, strain, sex, etc.), experimental characteristics (surgery, biomaterial, follow-up, etc.),

212 cell characteristics (cell type, culture conditions, etc.) and outcome characteristics (macroscopic 213 evaluation, histology and semi-quantitative histological scoring, etc.), as can be appreciated

214 from Supplementary Information 3. Various animal species were used including rabbit, dog, 215 sheep, pig, rat, horse, minipig, goat and macaques. A large range was found in animal age, e.g. 216 the age of rabbits ranged from 6 weeks to >2 years. Small animals were generally younger (in 217 the range of months) compared to larger animals (in the range of years). In many studies, no 218 detailed information was provided regarding the animal's absolute age, but merely e.g. adult or 219 mature.

The method for bone marrow stimulation was mostly subchondral drilling (142 studies), 221 where only 4 studies used microfracture. Defects were created at various locations (trochlea, 
222 condyles, femur and intercondylar fossa) and with diverse dimensions (e.g. for rabbits: 223 diameter 4-7 $\mathrm{mm}$ and depth 0.8-9 $\mathrm{mm}$ ).

224 Implanted biomaterials were prepared from natural (e.g. alginate and collagen), 225 synthetic (e.g. poly(lactic-coglycolic acid) and polycaprolactone) or mixtures of natural and 226 synthetic materials. In 27 studies biologics, such as bone morphogenetic protein 2 and 227 transforming growth factor beta, were loaded in the biomaterials. Different cell types were 228 applied, including chondrocytes, bone marrow-derived mesenchymal stem cells (BM-MSCs), 229 bone marrow-derived progenitor cells, synovium-derived stem cells (SD-MSCs), bone marrow230 derived mononuclear cells, adipose-derived stem cells, adipose-derived stromal vascular 231 fraction cells, endothelial progenitor cells, embryonic stem cells, umbilical cord blood stem 232 cells, fibroblasts, and periosteal cells, while in some studies undefined cell populations like 233 bone marrow aspirate concentrate were used. Cells were either seeded directly after 234 harvesting on biomaterials and implanted in the created defect or cultured in vitro to expand 235 and/or differentiate the cells, followed by seeding on biomaterials and implantation. In vitro 236 differentiation was performed with cells cultured in monolayer (without biomaterials), followed 237 by seeding of the cells onto the biomaterials and implantations, or by directly culturing the cells on biomaterials prior to implantation.

In most studies, short-term cartilage regeneration was investigated: the follow-up time was generally less than 6 months with a maximum follow-up of 12 months.

\subsection{Risk of bias assessment}

The methodological quality was assessed for all studies included in the meta-analysis except Chinese articles. The overview of the results in Figure 3 indicates a general lack of information regarding the experimental setup of the studies, limiting the assessment of the actual risk of bias. Please see Supplementary Information 5 for all scores per individual study.

In the assessed studies, details regarding the application and method of randomization

248 (Q1) were generally lacking. As a result, assessment of the actual risk of selection bias was practically impossible. Assessment of the actual risk of bias due to differences in baseline characteristics was difficult since no details regarding randomization were described. 
251 Differences may have been present in load-bearing between implantation sites (Q2.1) and age, 252 sex and weight of animals (Q2.2). In most studies, few differences were found between animals 253 at the moment of surgical intervention since animals were treated similarly (Q2.3). Details 254 regarding blinding of experimental conditions at the moment of implantation were generally 255 not provided, which may have resulted in bias (Q3). Random housing of animals was generally 256 not (well) described (Q4). Caregivers and/or investigators did not know which intervention each 257 animal received during the experiment (Q5). No details were presented regarding the random

258

259

260

261

262

263

264

265

266

267

268

269

270

271

272

273

274

275

276

277

278

279 selection of animals for outcome assessment (Q6). The method of blinding during analysis, however, was well described in most studies (Q7). Incomplete outcome data were identified or described in a few studies only, which resulted in studies with high risk of bias (Q8). Generally, most studies lacked reporting of important details and therefore adequate assessment of the actual risk of bias was difficult.

\subsection{Data synthesis}

Semi-quantitative histological scores were used as outcome data to compare biomaterials with cells (experimental group) and without cells (control group) and to address sub-questions related to the use of type of cells and culture conditions. An overview of all meta-analysis results is provided in Table 1; an overview of all raw data is given in Supplementary Information 6.

Data are presented as the effect (\%) with $95 \% \mathrm{Cl}$, where $100 \%$ cartilage regeneration represents healthy tissue and lower percentages indicate less regenerated cartilage tissue.

\subsubsection{Overall effect implantation of cellular and acellular biomaterials}

The meta-analysis indicates that implantation of cellular and acellular biomaterials resulted in $61.5 \%(95 \% \mathrm{Cl}:[58.5,64.5])$ and $43.0 \%$ (95\% Cl: $[40.0,46.0])$ cartilage regeneration, respectively. The addition of cells to biomaterials significantly improved cartilage regeneration by $18.6 \%$ (95\% Cl: $[15.2,22.0], \mathrm{p}<0.0001)$. An overview of results for each individual study is displayed in the forest plot (Supplementary Information 7), presenting improved cartilage regeneration by loading biomaterials with cells in 66 studies, similar cartilage regeneration in 30 
280 studies, and a negative effect on cartilage regeneration in 2 studies. The heterogeneity $\left(\mathrm{I}^{2}\right)$ for

281 the comparison between cellular and acellular biomaterials was very high $(99.4 \%[95 \% \mathrm{Cl}$

282 99.3\%; 99.4\%]).

283

284

\subsubsection{Stem cells and somatic cells}

No significant differences ( $p=0.622$ ) were found between biomaterials loaded with stem cells (61.5\% (95\% Cl: $[58.1,65.0]$ and somatic cells $(62.8 \%$ (95\% Cl: $[58.5,67.1])$.

\subsubsection{Cell type}

Biomaterials were loaded with various cell types. Subgroup analyses were only performed when subgroups consisted of more than five experimental groups in at least 3 studies. Seeding biomaterials with adipose-derived stem cells significantly decreased cartilage regeneration, while no other significant differences were observed (Table 1). Only for scaffolds seeded with adipose-derived stem cells (ADSCs), reduced cartilage regeneration was found $(56.3 \%(95 \% \mathrm{Cl}$ : $[49.9,62.6])$ compared to cellular scaffolds. However, cartilage regeneration using ADSCsseeded scaffolds still improved regeneration compared to acellular scaffolds.

\subsubsection{Cell manipulation}

298 Comparing differences in cartilage regeneration between biomaterials loaded with cells which were not cultured in vitro (implanted immediately after harvesting of cells) or were expanded and/or differentiated in vitro indicated that cell manipulation did not affect cartilage regeneration (Table 1).

\subsubsection{Sensitivity analyses}

304 To investigate the robustness of the meta-analysis, sensitivity analyses were performed regarding the overall effect of the addition of cells to biomaterials. The overall outcome effect for cellular scaffolds was not notably affected by the exclusion of studies (1) with assumptions (2) or written in Chinese (no risk of bias assessment performed). Also for acellular biomaterials, the exclusion of these studies had no effect on cartilage regeneration. 
$310 \quad 3.4 .5 \quad$ Publication bias

311 Publication bias was assessed for all studies included in the meta-analysis comparing cartilage 312 regeneration using acellular versus cellular biomaterials. Although the funnel plot (Fig. 4) is 313 rectangular in shape, no major asymmetry was observed, giving no indication for publication 314 bias (p-value 0.866). 


\section{Discussion}

316 Bone marrow stimulation can be applied to induce cartilage regeneration. Despite therapy, the

317 formed neotissue generally consists of fibrous cartilage, which lacks mechanical and biological

318 properties of native tissue [5]. Therefore, microfracture results in temporary clinical

319 improvement only [6]. To regenerate more durable cartilage tissue, regenerative medicine and

320 tissue engineering may offer a promising addition to bone marrow stimulation by the

321 implantation of scaffolds, which can act as a template to guide and stimulate cartilage

322 regeneration [3]. In a previous systematic review, the quality of newly formed cartilage in

323 animals was improved by the implantation of biomaterials after bone marrow stimulation,

324 which was further enhanced by loading biomaterials with biologics [8]. The aim of this

325 systematic review was (a) to provide a comprehensive and systematic overview of all current

326 literature regarding animal studies on cartilage regeneration using cellular versus acellular

327 biomaterials and to identify knowledge gaps, (b) to assess the efficacy of cartilage regeneration

328 using cellular versus acellular biomaterials and to investigate the effect of various parameters

329 (i.e. stem/somatic cells, cell source, cell culture conditions), (c) to gain insight in the

330 methodological quality of animal studies, and (d) to improve the design of future animal models

331 and eventually clinical trials.

332 In animal studies, the implantation of cellular biomaterials in animal models significantly

333 improved cartilage regeneration by $18.6 \%$ compared to acellular biomaterials. Seeding of cells

334 is a major component of the tissue engineering paradigm, which may stimulate healing by the

335 production of many bioactive components. Therefore, the addition of cells to biomaterials

336 enhanced the regenerative process [48]. The heterogeneity $\left(I^{2}\right)$ for the main research question

337 and subgroup analyses was very high. Results should therefore be interpreted with caution,

338 especially for subgroup analyses with a limited number of studies. Further clinical studies are

339 required to assess the potential beneficial effect of cellular biomaterials versus acellular

340 biomaterials in patients. Marcacci et al. [49] published promising results of a multicenter clinical

341 phase III retrospective cohort study in which patients were treated with an implant consisting

342 of autologous chondrocytes grown on Hyalograft C, a hyaluronic acid derivative, with a 3-year 
343 follow-up. Assessment indicated major clinical improvements and hyaline-like cartilage for the 344 majority of biopsies.

345 In a subgroup analysis, no significant differences were found between somatic cells and 346 stem cells. Differences were found between various cell types. Adipose-derived stem cells 347 (ADSCs) reduced cartilage regeneration in the subgroup analysis. However, cartilage 348 regeneration using biomaterials seeded with ADSCs was still superior to biomaterials without 349 cells. As compared to other cell types, the origin of ADSCs from fatty tissue may have resulted 350 in significantly reduced cartilage regeneration compared to cells derived from cartilage or 351 subchondral bone. MSCs and chondrocytes have distinct advantages. MSCs are not limited by 352 donor-site morbidity and matrix production after expansion in vitro [50], can be harvested from 353 numerous sources, maintain their multipotency after expansion in vitro, can differentiate into 354 chondrocytes that produce cartilage matrix and may suppress proinflammatory cytokines by 355 their immunoregulatory properties. Chondrocytes on the other hand do not terminally 356 differentiate after chondrogenic differentiation, which results in bone formation [50], and are 357 more easy to manipulate [51]. In clinical trials, the addition of MSCs or chondrocytes to 358 biomaterials resulted in comparable cartilage regeneration $[52,53]$. In this study no subgroup analysis was performed to investigate the culture of cell-loaded scaffolds in bioreactors.

360 Bernhard et al. described the beneficial effects of culturing cell-loaded scaffolds in bioreactors with mechanical loading protocols, as these scaffolds more closely resembled the native compressive properties of cartilage tissue and as the applied force steered the location and alignment of cartilage matrix deposition by chondrocytes [50].

Study characteristics showed a large heterogeneity between studies due to differences in animal model, performed surgery, implanted biomaterial and follow-up period. To reduce the influence of possible confounding parameters, we excluded studies using healthy animals in which created defects were not filled during the first surgery and osteoarthritis animal models, despite their greater relevance for future applications to treat patients with osteoarthritis.

Various outcome measures were used to investigate cartilage regeneration, including MRI, macroscopic and histological evaluation (more extensively discussed in [8]. We selected data from semi-quantitative histological scoring systems as outcome measure, because 
372 histological scores are frequently used and allow for quantitative comparisons between studies.

373 However, different scoring systems are available (extensively reviewed by Rutgers et al. [37])

374 that assess different processes, e.g. cartilage regeneration only, cartilage and subchondral bone

375 regeneration, and additional biomaterial degradation. Not discriminating between these

376 parameters may be considered as a limitation, but usage of all scoring systems may provide an

377 extensive and complete overview of all aspects affecting the regenerative process. Additionally,

378 evaluation of cartilage regeneration using semi-quantitative histological scoring may still be

379 observer-dependent and subjective, possibly inducing observer (detection) bias. Therefore, it

380 may be better to combine histological scores with biochemical parameters and biomechanical

381 properties, but the ideal combination of outcome parameters remains unknown [37].

382 The methodological quality assessment was performed to evaluate the experimental

383 designs and reliability of the results of included studies. The methodological quality (internal

384 validity) is of great importance since a low methodological quality may result in an

385 overestimation or underestimation of the effect size [54]. No studies were included in or

386 excluded from the meta-analysis based on methodological quality assessment results.

387 Generally, the possibility of assessing the actual risk of bias was limited due to the absence of 388 important details regarding the experimental set-up in most studies and method of 389 randomization. It may be that the animal studies were performed well, but that experimental 390 designs were only reported poorly [55]. For the analysis of the histological sections, however,

391 most studies described that sections were randomized and that outcome assessors were 392 blinded. Detection/observer bias may be introduced in case blinding was not performed and 393 can result in an overestimation of the actual effect of the therapy [56]. The overall validity of 394 the study results may be impaired by bias due to the lack of blinding and randomization [57, 395 58]. Reporting of animal studies may be improved by using standardized protocols, including 396 the ARRIVE guidelines [59] or golden standard publication checklist [60].

A high translational value of animal studies is crucial to take treatments forward to 398 clinical practice. Therefore, validated and predictive animal models are required. Many 399 challenges and limitations are associated with the use of animal models for cartilage defects. 400 Chu et al. [61] and Ahern et al. [62] extensively described strengths and shortcomings of 
401 different animal models related to e.g. joint size, cartilage thickness, defect size, intrinsic 402 healing potential and animal maturity, in comparison to lesions in clinical studies. In most 403 animal experiments, the follow-up period was maximally 6 months, while in patients clinical 404 improvements are generally observed up to 1.5 - 3 years after microfracture surgery $[63,64]$. 405 The translational value and considerations to select animal models were extensively discussed 406 before [8].

407 Improved reporting of animal studies is required in future studies and studies should 408 strive to resemble the clinical situation to facilitate translation. For clinical application of new 409 regenerative medicine and tissue engineering strategies, including the use of biomaterials, 410 biologics and cells, the effectiveness needs to be proven both in animal models and clinical 411 studies [65]. Moreover, the cost-effectiveness of new interventions in clinical practice may be 412 assessed using early health economic models [66]. Considerations for the addition of cells to 413 biomaterials are of great importance and limitations (including donor-site morbidity, cell 414 culture costs, regulatory issues, limited off the shelf availability, and potential multiple-stage 415 surgical procedures [8, 67]) should be weighed against potentially superior cartilage 416 regeneration by applying cellular biomaterials. Difficulties in controlling cell culture and the 417 development of novel materials stimulating tissue regeneration may justify the use of acellular 418 biomaterials. Future research focusing on biomaterials properties, source and manipulation of 419 cells, and possibly patient profiling, may allow selection of the best treatment for each 420 individual patient [68]. 


\section{Conclusion}

422 This systematic review and meta-analysis provides an extensive overview of all animal studies

423 applying regenerative medicine and tissue engineering approaches to regenerate articular 424 cartilage by implantation of cellular versus acellular biomaterials after applying bone barrow 425 stimulation. Cartilage regeneration was more effective by implantation of cellular biomaterials 426 compared to acellular biomaterials. This study together with a previous study on the beneficial 427 properties of scaffolds and growth factors implies that all components of the tissue engineering 428 paradigm can be valuable for improved regeneration of articular cartilage.

\section{Manuscript information and declarations}

\section{Acknowledgements:}

432 We thank Jie An (Department of Biomaterials, Radboud Institute for Molecular Life Sciences, 433 Radboud university medical center) and Chunling Tang (Department of Tumor Immunology, 434 Radboud Institute for Molecular Life Sciences, Radboud university medical center) for their 435 contribution to the paper. Gerrie Hermkens from the Radboud university medical center library 436 is greatly acknowledged for help retrieving full-text studies.

437

\section{Supplementary Information}

439 Raw data are provided in Supplementary Information 6. 


\section{References}

441 1. Swieszkowski W, Tuan BH, Kurzydlowski KJ, Hutmacher DW. Repair and regeneration of

$442 \quad$ osteochondral defects in the articular joints. Biomol Eng 2007; 24: 489-495.

443 2. Buckwalter JA, Saltzman C, Brown T. The impact of osteoarthritis: implications for research. Clin Orthop Relat Res 2004; 427 Suppl: S6-15.

3. Cucchiarini M, Madry H, Guilak F, Saris DB, Stoddart MJ, Koon Wong M, Roughley P. A vision on the future of articular cartilage repair. Eur Cell Mater 2014; 27: 12-16.

4. Steadman JR, Rodkey WG, Rodrigo JJ. Microfracture: surgical technique and rehabilitation to treat chondral defects. Clin Orthop Relat Res 2001; 391 Suppl: S362369.

5. Dai L, He Z, Zhang X, Hu X, Yuan L, Qiang M, Zhu J, Shao Z, Zhou C, Ao Y. One-step repair

6. Saris D, Price A, Widuchowski W, Bertrand-Marchand M, Caron J, Drogset JO, Emans P, for cartilage defects in a rabbit model: a technique combining the perforated decalcified cortical-cancellous bone matrix scaffold with microfracture. Am J Sports Med 2014; 42: 583-591.

459 Podskubka A, Tsuchida A, Kili S, Levine D, Brittberg M; SUMMIT study group. Matrixapplied characterized autologous cultured chondrocytes versus microfracture: two-year follow-up of a prospective randomized trial. Am J Sports Med 2014; 42: 1384-1394.

7. Seo SJ, Mahapatra C, Singh RK, Knowles JC, Kim HW. Strategies for osteochondral repair: focus on scaffolds. J Tissue Eng 2014; 5: 2041731414541850.

9. Ahn JH, Lee TH, Oh JS, Kim SY, Kim HJ, Park IK, Choi BS, Im GI. Novel hyaluronate-

11. Christensen BB, Foldager CB, Hansen OM, Kristiansen AA, Le DQ, Nielsen AD, Nygaard $\mathrm{JV}$, Bünger $\mathrm{CE}$, Lind $\mathrm{M}$. A novel nano-structured porous polycaprolactone scaffold improves hyaline cartilage repair in a rabbit model compared to a collagen type I/III scaffold: in vitro and in vivo studies. Knee Surg Sports Traumatol Arthrosc 2012; 20: 1192-1204.

12. Araki S, Imai S, Ishigaki H, Mimura T, Nishizawa K, Ueba H, Kumagai K, Kubo M, Mori K, Ogasawara K, Matsusue Y. Improved quality of cartilage repair by bone marrow mesenchymal stem cells for treatment of an osteochondral defect in a cynomolgus macaque model. Acta Orthop 2015; 86: 119-126.

$Y$, Minami A. Repair of articular cartilage defects with a novel injectable in situ forming material in a canine model. J Biomed Mater Res A 2012; 100: 180-187. 
483 14. Wakitani S, Goto T, Pineda SJ, Young RG, Mansour JM, Caplan Al, Goldberg VM.

484

485

486

487

488

489

490

491

492

493

494

495

496

497

498

499

500

501

502

503

504

505

506

507

508

509

510

511

512

513

514

515

516

517

518

519

520

521

522

523

524

525 Mesenchymal cell-based repair of large, full-thickness defects of articular cartilage. J Bone Joint Surg Am 1994; 76: 579-592.

15. Pei M, He F, Boyce BM, Kish VL. Repair of full-thickness femoral condyle cartilage defects using allogeneic synovial cell-engineered tissue constructs. Osteoarthritis Cartilage 2009; 17: 714-722.

16. Lee JC, Min HJ, Park HJ, Lee S, Seong SC, Lee MC. Synovial membrane-derived mesenchymal stem cells supported by platelet-rich plasma can repair osteochondral defects in a rabbit model. Arthroscopy 2013; 29: 1034-1046.

17. Shimomura K, Moriguchi Y, Ando W, Nansai R, Fujie H, Hart DA, Gobbi A, Kita K, Horibe $\mathrm{S}$, Shino K, Yoshikawa H, Nakamura N. Osteochondral repair using a scaffold-free tissueengineered construct derived from synovial mesenchymal stem cells and a hydroxyapatite-based artificial bone. Tissue Eng Part A 2014; 20: 2291-2304.

18. Xie XT, Wang Y, Zhao CJ, Guo SC, Liu S, Jia WT, Tuan RS, Zhang C. Comparative evaluation of MSCs from bone marrow and adipose tissue seeded in PRP-derived scaffold for cartilage regeneration. Biomaterials 2012; 33: 7008-7018.

19. Masuoka K, Asazuma T, Hattori H, Yoshihara Y, Sato M, Matsumra K, Matsui T, Takase B, Nemoto K, Ishihara M. Tissue engineering of articular cartilage with autologous cultured adipose tissue-derived stromal cells using atelocollagen honeycomb-shaped scaffold with a membrane sealing in rabbits. J Biomed Mater Res B Appl Biomater 2006; 79B: 2534.

20. Kang HJ, Peng J, Lu SB, Liu SY, Zhang L, Huang JX, Sui X, Zhao B, Wang A, Xu W, Luo Z, Guo Q. In vivo cartilage repair using adipose-derived stem cell-loaded decellularized cartilage ECM scaffolds. J Tissue Eng Regen Med 2014; 8: 442-453.

21. Perka C, Schultz O, Spitzer RS, Lindenhayn K. The influence of transforming growth factor beta 1 on mesenchymal cell repair of full-thickness cartilage defects. J Biomed Mater Res 2000; 52: 543-552.

22. Schagemann JC, Erggelet C, Chung HW, Lahm A, Kurz H, Mrosek EH. Cell-laden and cellfree biopolymer hydrogel for the treatment of osteochondral defects in a sheep model. Tissue Eng Part A 2009; 15: 75-82.

23. Yan H, Yu CL. Repair of full-thickness cartilage defects with cells of different origin in a rabbit model. Arthroscopy 2007; 23: 178-187.

24. Chung JY, Song M, Ha CW, Kim JA, Lee CH, Park YB. Comparison of articular cartilage repair with different hydrogel-human umbilical cord blood-derived mesenchymal stem cell composites in a rat model. Stem Cell Res Ther 2014; 5: 39-52.

25. Cheng A, Kapacee Z, Hardingham T, Lucas R, Kimber S. Cartilage Repair using Human Embryonic Stem Cell-derived Chondroprogenitors. Stem Cells Transl Med 2014; 3: 12871294.

26. Betsch M, Thelen S, Santak L, Herten M, Jungbluth P, Miersch D, Hakimi M, Wild M. The role of erythropoietin and bone marrow concentrate in the treatment of osteochondral defects in mini-pigs. PLoS One 2014; 9: e92766.

27. Getgood A, Henson F, Skelton C, Herrera E, Brooks R, Fortier LA, Rushton N. The augmentation of a collagen/glycosaminoglycan biphasic osteochondral scaffold with 
platelet-rich plasma and concentrated bone marrow aspirate for osteochondral defect repair in sheep: a pilot study. Cartilage 2012; 3: 351-363.

28. Guo X, Park H, Young S, Kretlow JD, van den Beucken JJ, Baggett LS, Tabata Y, Kasper FK, Mikos AG, Jansen JA. Repair of osteochondral defects with biodegradable hydrogel composites encapsulating marrow mesenchymal stem cells in a rabbit model. Acta Biomater 2010; 6: 39-47.

29. Dorotka R, Bindreiter U, Macfelda K, Windberger U, Nehrer S. Marrow stimulation and chondrocyte transplantation using a collagen matrix for cartilage repair. Osteoarthritis Cartilage 2005; 13: 655-664.

30. Sosio C, Di Giancamillo A, Deponti D, Gervaso F, Scalera F, Melato M, Campagnol M, Boschetti F, Nonis A, Domeneghini C, Sannino A, Peretti GM. Osteochondral repair by a novel interconnecting collagen-hydroxyapatite substitute: a large-animal study. Tissue Eng Part A 2015; 21: 704-715.

31. Necas A, Plánka L, Srnec R, Crha M, Hlucilová J, Klíma J, Starý D, Kren L, Amler E, Vojtová L, Jancár J, Gál P. Quality of newly formed cartilaginous tissue in defects of articular surface after transplantation of mesenchymal stem cells in a composite scaffold based on collagen I with chitosan micro- and nanofibres. Physiol Res 2010; 59: 605-614.

32. de Vries RB, Buma P, Leenaars M, Ritskes-Hoitinga M, Gordijn B. Reducing the number of laboratory animals used in tissue engineering research by restricting the variety of animal models. Articular cartilage tissue engineering as a case study. Tissue Eng Part B Rev 2012; 18: 427-235.

33. Leenaars $M$, Hooijmans $C R$, van Veggel $N$, ter Riet $G$, Leeflang $M$, Hooft $L$, van der Wilt GJ, Tillema A, Ritskes-Hoitinga M. A step-by-step guide to systematically identify all relevant animal studies. Lab Anim 2012; 46: 24-31.

34. Sloff $M$, Simaioforidis $V$, de Vries R, Oosterwijk E, Feitz W. Tissue engineering of the bladder-reality or myth? A systematic review. J Urol 2014; 192: 1035-1042.

35. Hooijmans CR, Tillema A, Leenaars M, Ritskes-Hoitinga M. Enhancing search efficiency by means of a search filter for finding all studies on animal experimentation in PubMed. Lab Anim 2010; 44: 170-175.

36. de Vries RB, Hooijmans CR, Tillema A, Leenaars M, Ritskes-Hoitinga M. A search filter for increasing the retrieval of animal studies in Embase. Lab Anim 2011; 45: 268-270.

37. Hooijmans CR, Rovers MM, de Vries RB, Leenaars M, Ritskes-Hoitinga M, Langendam MW. SYRCLE's risk of bias tool for animal studies. BMC Med Res Methodol 2014; 14: 4352.

38. Rutgers M, van Pelt MJ, Dhert WJ, Creemers LB, Saris DB. Evaluation of histological scoring systems for tissue-engineered, repaired and osteoarthritic cartilage. Osteoarthritis Cartilage 2010; 18: 12-23.

39. Hozo SP, Djulbegovic B, Hozo I. Estimating the mean and variance from the median, range, and the size of a sample. BMC Med Res Methodol 2005; 5: 13-23.

40. Higgins JPT, Green, S. Cochrane handbook for systematic reviews of interventions 2011. London: The Cochrane Collaboration.

41. R Core Team. R: A language and environment for statistical computing. R Foundation for Statistical Computing 2011, Vienna, Austria. http://www.R-project.org/. 
569 42. Schwarzer G. meta: General package for meta-analysis. R package version 4.1-0.

570

571

572

573

574

575

576

577

578

579

580

581

582

583

584

585

586

587

588

589

590

591

592

593

594

595

596

597

598

599

600

601

602

603

604

605

606

607

608

609

610 http://CRAN.R-project.org/package=meta.

43. Thompson SG, Sharp SJ. Explaining heterogeneity in meta-analysis: a comparison of methods. Stat Med 1999; 18: 2693-2708.

44. Higgins JP, Thompson SG, Deeks JJ, Altman DG. Measuring inconsistency in metaanalyses. BMJ 2003; 327: 557-560.

45. Xie A, Nie L, Shen G, Cui Z, Xu P, Ge H, Tan Q. The application of autologous plateletrich plasma gel in cartilage regeneration. Mol Med Rep 2014; 10: 1642-1648.

46. Yao X, Ma X, Zhang Z. Chondrocyte allografts for repair of full-thickness defects in the condylar articular cartilage of rabbits. Chin J Dent Res 2000; 3: 24-30.

47. Zhou M, Yu D. Cartilage tissue engineering using PHBV and PHBV/Bioglass scaffolds. Mol Med Rep 2014; 10: 508-514.

48. Wang M, Yuan Z, Ma N, Hao C, Guo W, Zou G, Zhang Y, Chen M, Gao S, Peng J, Wang A, Wang $Y$, Sui $X, X u$ W, Lu S, Liu S, Guo Q. Advances and prospects in stem cells for cartilage regeneration. Stem Cells Int 2017; 4130607.

49. Marcacci $M$, Berruto $M$, Brocchetta D, Delcogliano A, Ghinelli D, Gobbi A, Kon E, Pederzini L, Rosa D, Sacchetti GL, Stefani G, Zanasi S. Articular cartilage engineering with Hyalograft C: 3-year clinical results. Clin Orthop Relat Res 2005; 435: 96-105.

50. Bernhard JC, Vunjak-Novakovic G. Should we use cells, biomaterials, or tissue engineering for cartilage regeneration? Stem Cell Res Ther 2016; 7: 56-65.

51. Deng Z, Jin J, Zhao J, Xu H. Cartilage defect treatments: with or without cells? Mesenchymal stem cells or chondrocytes? Traditional or matrix-assisted? A systematic review and meta-analyses. Stem Cells Int 2016; 9201492.

52. Nejadnik H, Hui JH, Feng Choong EP, Tai BC, Lee EH. Autologous bone marrow-derived mesenchymal stem cells versus autologous chondrocyte implantation: an observational cohort study. Am J Sports Med 2010; 38: 1110-1116.

53. Lee KB, Wang VT, Chan $\mathrm{YH}$, Hui JH. A novel, minimally-invasive technique of cartilage repair in the human knee using arthroscopic microfracture and injections of mesenchymal stem cells and hyaluronic acid--a prospective comparative study on safety and short-term efficacy. Ann Acad Med Singapore 2012; 41: 511-517.

54. Higgins JP, Altman DG, Gotzsche PC, Juni P, Moher D, Oxman AD, Savovic J, Schulz KF, Weeks L, Sterne JA; Cochrane Bias Methods Group; Cochrane Statistical Methods Group. The Cochrane Collaboration's tool for assessing risk of bias in randomised trials. BMJ 2011; 343: d5928.

55. Hooijmans CR, de Vries RB, Rovers MM, Gooszen HG, Ritskes-Hoitinga M. The effects of probiotic supplementation on experimental acute pancreatitis: a systematic review and meta-analysis. PLoS One 2012; 7: e48811.

56. Bello S, Krogsboll LT, Gruber J, Zhao ZJ, Fischer D, Hrobjartsson A. Lack of blinding of outcome assessors in animal model experiments implies risk of observer bias. J Clin Epidemiol 2014; 67: 973-983.

57. Bebarta V, Luyten D, Heard K. Emergency medicine animal research: does use of randomization and blinding affect the results? Acad Emerg Med 2003; 10: 684-687. 
611 58. Hirst JA, Howick J, Aronson JK, Roberts N, Perera R, Koshiaris C, Heneghan C. The need

612

613

614

615

616

617

618

619

620

621

622

623

624

625

626

627

628

629

630

631

632

633

634

635

636

637

638

639

640

641

642

643

644 for randomization in animal trials: an overview of systematic reviews. PLoS One 2014; 9: e98856.

59. Kilkenny C, Browne WJ, Cuthi I, Emerson M, Altman DG. Improving bioscience research reporting: the ARRIVE guidelines for reporting animal research. PLoS Biol 2012; 41: 2731.

60. Hooijmans C, de Vries R, Leenaars M, Ritskes-Hoitinga M. The Gold Standard Publication Checklist (GSPC) for improved design, reporting and scientific quality of animal studies GSPC versus ARRIVE guidelines. Lab Anim 2011; 45: 61.

61. Chu CR, Szczodry M, Bruno S. Animal models for cartilage regeneration and repair. Tissue Eng Part B Rev 2010; 16: 105-115.

62. Ahern BJ, Parvizi J, Boston R, Schaer TP. Preclinical animal models in single site cartilage defect testing: a systematic review. Osteoarthritis Cartilage 2009; 17: 705-713.

63. Hoemann CD, Chen G, Marchand C, Tran-Khanh N, Thibault M, Chevrier A, Sun J, Shive MS, Fernandes MJ, Poubelle PE, Centola M, El-Gabalawy H. Scaffold-guided subchondral bone repair: implication of neutrophils and alternatively activated arginase-1+ macrophages. Am J Sports Med 2010; 38: 1845-1856.

64. van der Linden MH, Saris D, Bulstra SK, Buma P. Treatment of cartilaginous defects in the knee: recommendations from the Dutch Orthopaedic Association. Ned Tijdschr Geneeskd 2013; 157: A5719.

65. Cousin MA, Greenberg AJ, Koep TH, Angius D, Yaszemski MJ, Spinner RJ, Windebank AJ. The Value of Systematic Reviews in Estimating the Cost and Barriers to Translation in Tissue Engineering. Tissue Eng Part B Rev 2016; 22: 430-437.

66. de Windt TS, Sorel JC, Vonk LA, Kip MM, ljzerman MJ, Saris DB. Early health economic modelling of single-stage cartilage repair. Guiding implementation of technologies in regenerative medicine. J Tissue Eng Regen Med 2016; in press.

67. Efe T, Theisen C, Fuchs-Winkelmann S, Stein T, Getgood A, Rominger MB, Paletta JR, Schofer MD. Cell-free collagen type I matrix for repair of cartilage defects-clinical and magnetic resonance imaging results. Knee Surg Sports Traumatol Arthrosc 2012; 20: 1915-1922.

68. Kon E, Roffi A, Filardo G, Tesei G, Marcacci M. Scaffold-based cartilage treatments: with or without cells? A systematic review of preclinical and clinical evidence. Arthroscopy 2015; 31: 767-775. 


\section{Figure 1}

Figure 1

Illustration of articular cartilage regeneration by implantation of cellular and acellular biomaterials after applying bone marrow stimulation. The figure was adapted from Pot et al. [8]. 


\section{Cartilage regeneration by implantation of acellular or cell-laden biomaterials}

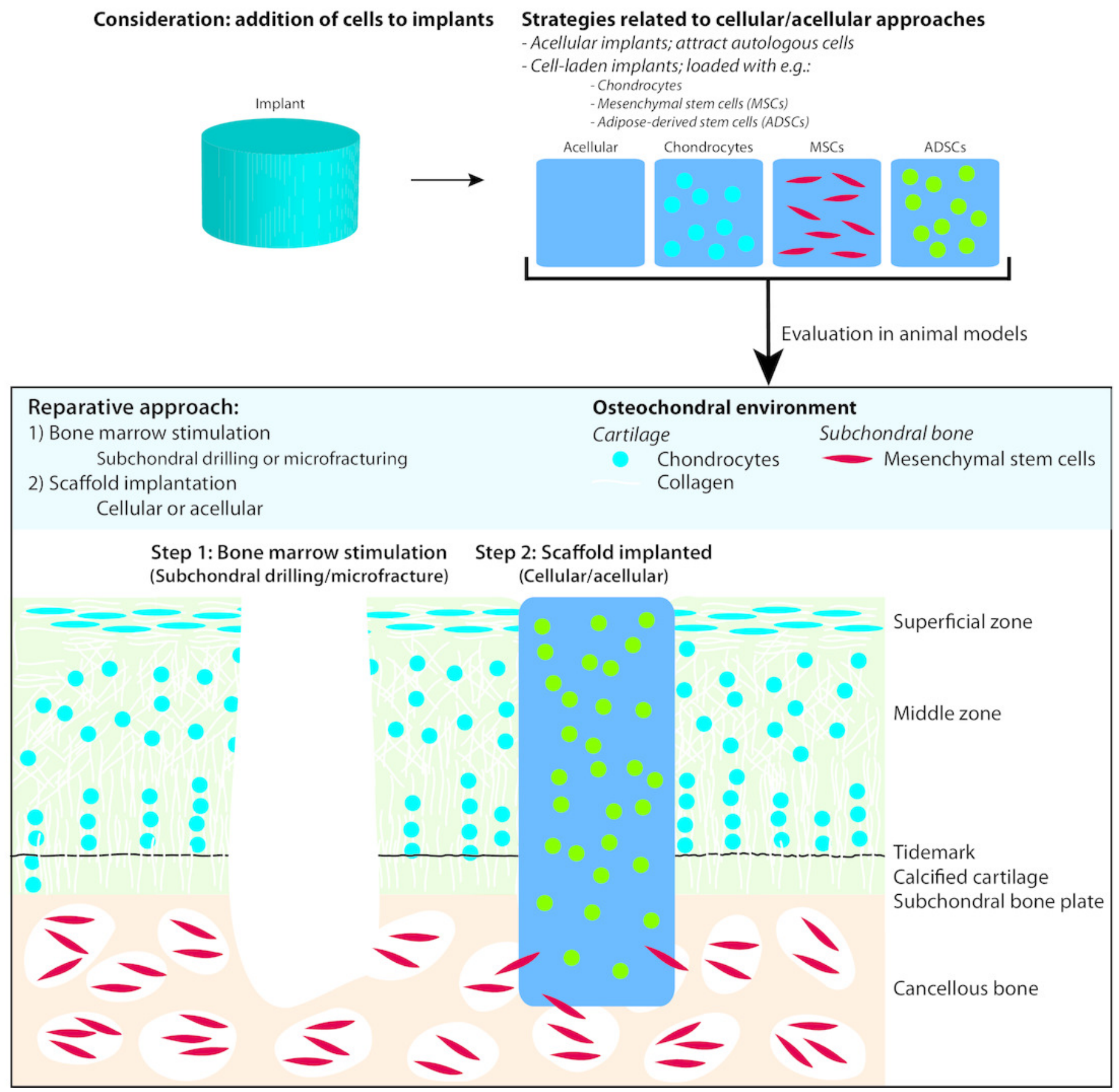




\section{Figure 2}

Figure 2

PRISMA (Preferred Reporting Items for Systematic Reviews and Meta-analysis) flowchart of the systematic search of literature. Of the 117 studies included for the meta-analysis, a risk of bias assessment was performed for 101 studies, excluding Chinese articles. 


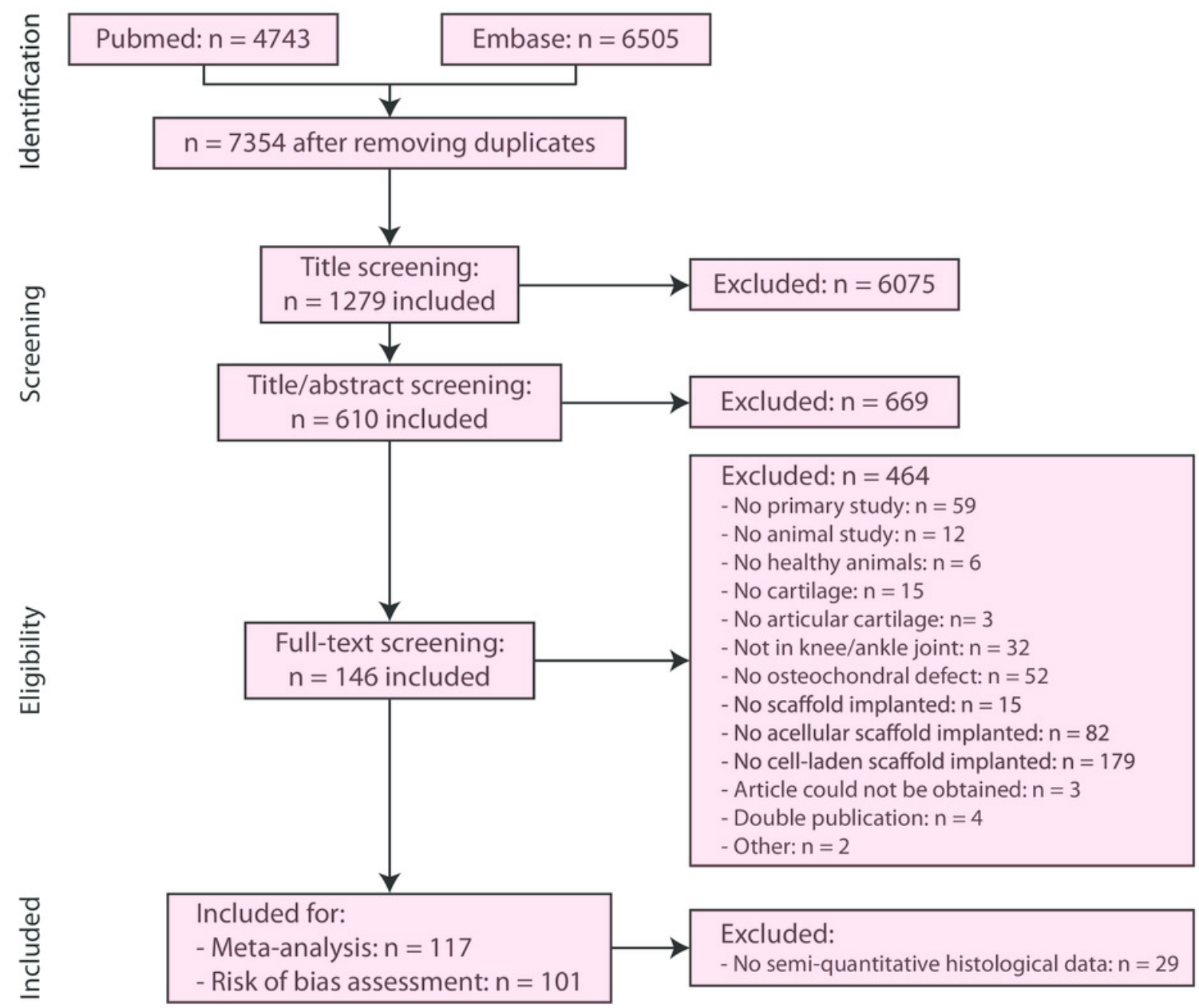




\section{Figure 3}

Figure 3

Results of the risk of bias analysis. Low, unknown or high risk of bias are presented in green, orange and red, respectively, where the percentages indicate the percentage of studies scoring low, unknown or high risk of bias of the total number of investigated studies per question. Low risk of bias was mainly found for addressing incomplete outcome data and baseline characteristics at the moment of surgical intervention. Unknown risk of bias was generally the result of limited details described in the studies regarding the experimental set-up. High risk of bias was only occasionally scored. Questions 4-6 are not depicted graphically, but are described and explained in Supplementary Information 4. 
1. Randomization: Was the allocation sequence adequately generated and applied?

2.1. Baseline characteristics: Number of conditions/animal? 2.2. Baseline characteristics: Dividing animal characteristics over conditions? -

2.3: Baseline characteristics: Difference at moment of surgical intervention? 3. Implantation concealment: Was the allocation adequately concealed? -

7. Analysis blinding: Was the outcome assessor blinded? 8. Incomplete outcome data: Were incomplete outcome data adequately addressed? -

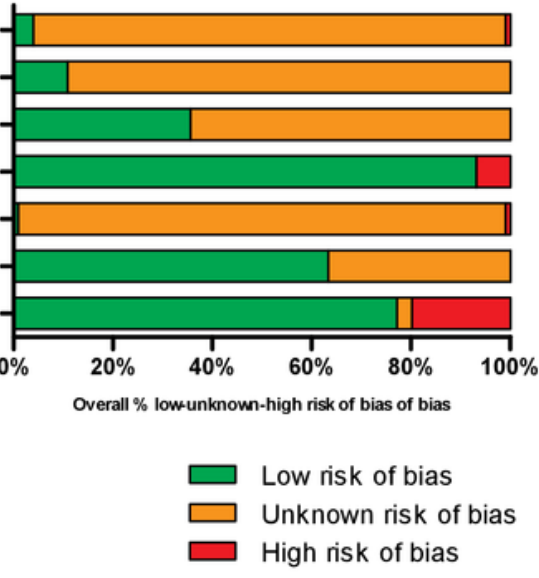




\section{Figure 4}

Figure 4

Funnel plot of the studies included in the meta-analysis comparing cartilage regeneration using cell-laden and acellular biomaterials. No substantial asymmetry was found.

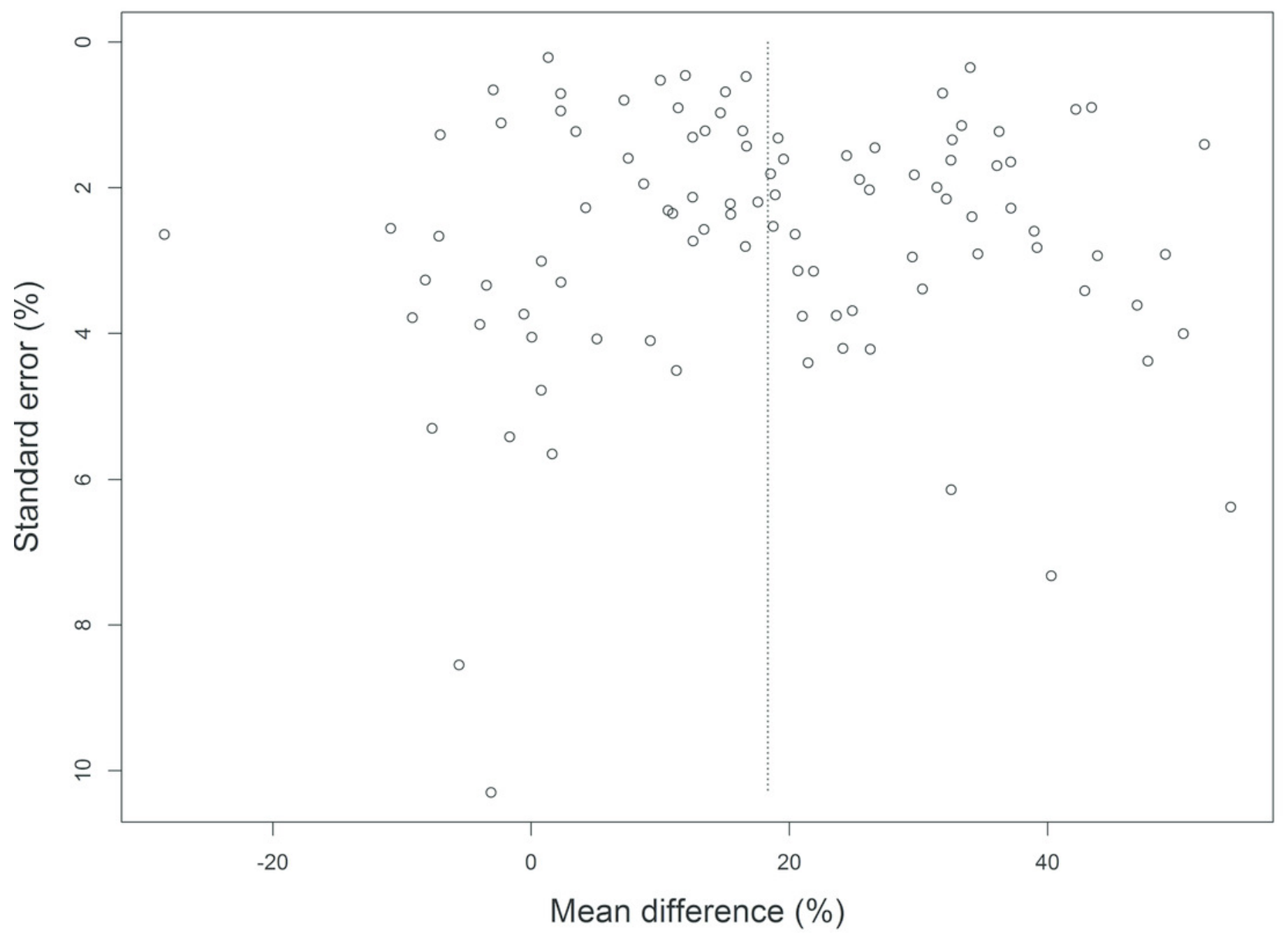




\section{Table $\mathbf{1}$ (on next page)}

Table 1

Overview meta-analysis results; the effect on cartilage regeneration of (1) the addition of cells to biomaterials, (2) loading of stem cells vs. somatic cells, (3) loading of specific cell types, e.g. chondrocytes vs. all cells except chondrocytes, and (4) culture conditions. The total number of studies and number of groups included in the meta-analysis are depicted (studies may have $>1$ experimental group, no. of studies/groups). Results are presented on a $100 \%$ cartilage regeneration scale, where $100 \%$ indicates 'maximum' cartilage regeneration. The addition of cells to biomaterials significantly improved cartilage regeneration compared to acellular biomaterials. The use of stem cells or somatic cells resulted in comparable cartilage regeneration. Cartilage regeneration was significantly lower for biomaterials seeded with adipose-derived stem cells compared to other cell types. Cartilage regeneration was not affected by the method of cell manipulation. 


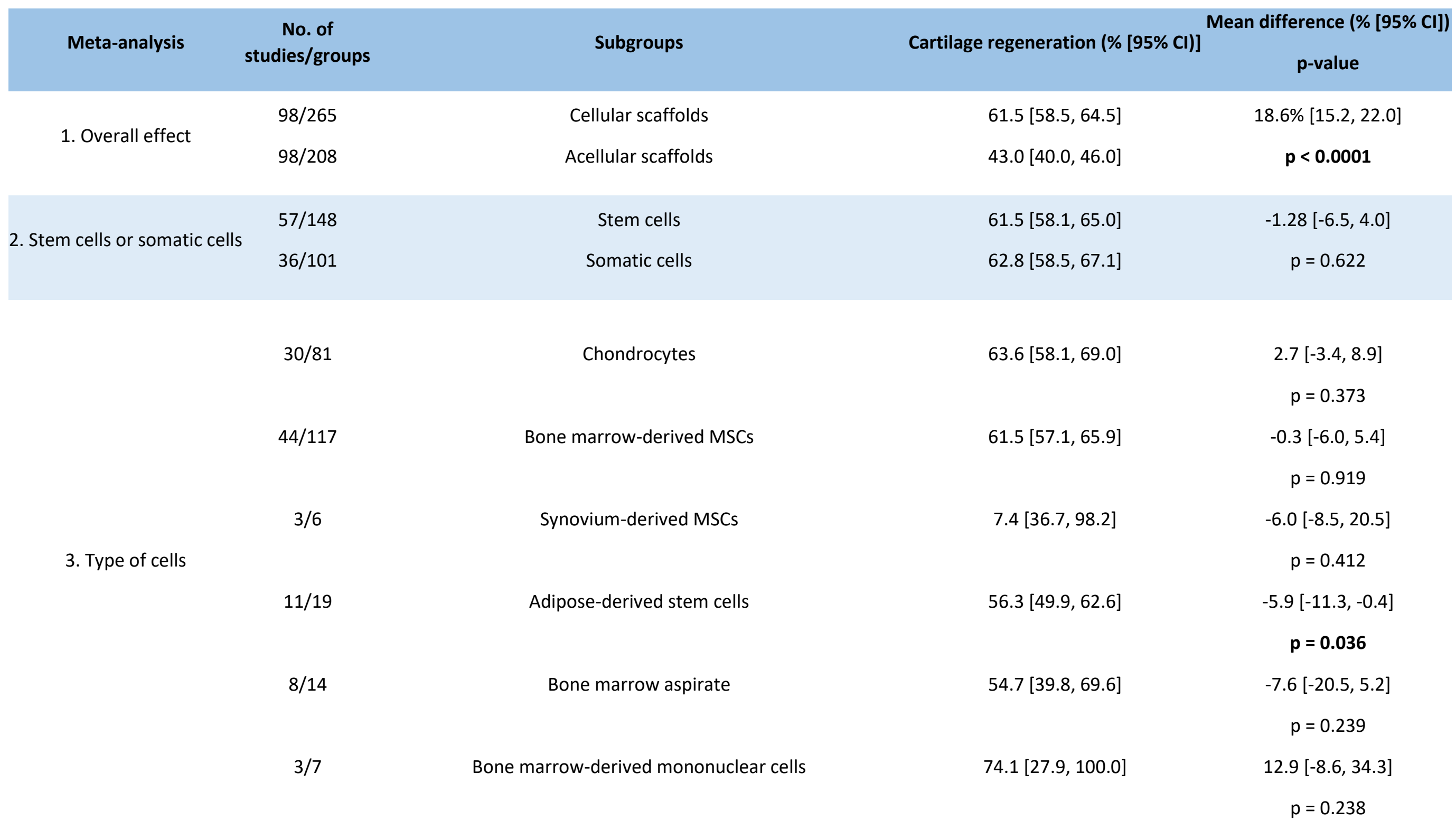




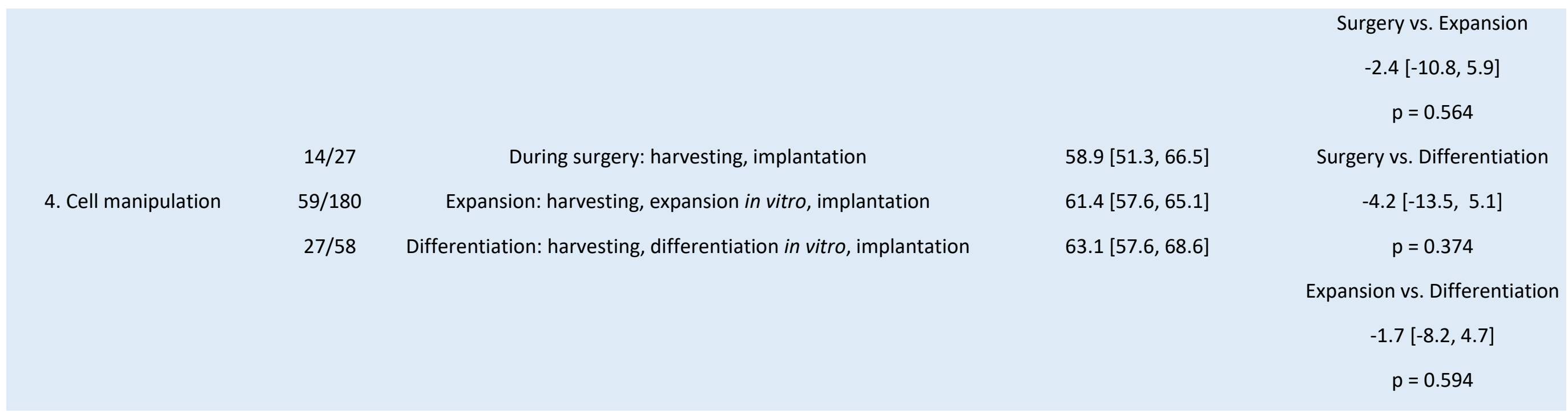

